\title{
Subtree-based XML Storage and XPath Processing
}

\author{
Ki Hoon Shin and Hyunchul Kang \\ School of Computer Science and Engineering, Chung-Ang University \\ Seoul, 156-756, Korea \\ [e-mail: koronya@gmail.com, hckang@cau.ac.kr] \\ *Corresponding author: Hyunchul Kang \\ Received August 27, 2010; revised September 5, 2010; accepted September 7, 2010; \\ published October 30, 2010
}

\begin{abstract}
The state-of-the-art techniques of storing XML data, modeled as an XML tree, are node-based in the sense that they are centered around XML node labeling and the storage unit is an XML node. In this paper, we propose a generalization of such techniques so that the storage unit is an XML subtree that consists of one or more nodes. Despite several advantages with such generalization, a major problem would be inefficiency in XPath processing where the stored subtrees are to be parsed on the fly in order for the nodes inside them to be accessed. We solve this problem, proposing a technique whereby no parsing of the subtrees involved in XPath processing is needed at all unless they contain the nodes of the final query result. We prove that the correctness of XPath processing is guaranteed with our technique. Through implementation and experiments, we also show that the overhead of our technique is acceptable.
\end{abstract}

Keywords: XML storage, XPath processing, XML node labeling, XML tree

This research was supported by the Chung-Ang University Research Grants in 2010.

DOI: $10.3837 /$ tiis.2010.10.0010 


\section{Introduction}

Over the last decade, XML has been established as the standard for data representation and exchange on the Internet. One of the fundamental problems in XML data processing is to store XML data and process queries against it. The state-of-the-art technique of storing XML data has been centered around XML node labeling, described as follows: An XML document is modeled as a tree. Fig. 1-(a) and (b) shows a sample XML data and its XML tree. Three types of XML nodes are shown: element, attribute, and text. Fig. 2 shows the XML tree of Fig. 1-(b) where each node is labeled, for example, with the well-known range numbering scheme of [1] and the nodes with the same element tag/attribute name are distinguished with a subscript number (e.g., $b_{1}, b_{2}$ ) just for exposition below. Each label denotes the (begin:end) range and the level of a node. For example, $(2: 21,2)$ labeled to $b_{1}$ denotes that the (begin:end) range assigned to $b_{1}$ is $[2,21]$ and that the level of $b_{1}$ in the tree is 2 . Those labels are for structural join [1][2][3][4] in XPath processing. For any two nodes $n_{1}$ and $n_{2}$, their structural relationship (ancestor-descendant or parent-child) can be determined with their labels. If the range of $n_{1}$ contains that of $n_{2}, n_{1}$ is an ancestor of $n_{2}$. If the levels of an ancestor-descendant pair differs by 1, one is the parent of the other. In Fig. 2, for example, $b_{1}$ is an ancestor of $d_{2}$ because the range of $b_{1}(2: 21)$ contains that of $d_{2}(16: 19)$ whereas $b_{1}$ is not an ancestor of $d_{4}$ because the range of $b_{1}$ (2:21) does not contain that of $d_{4}$ (36:39).

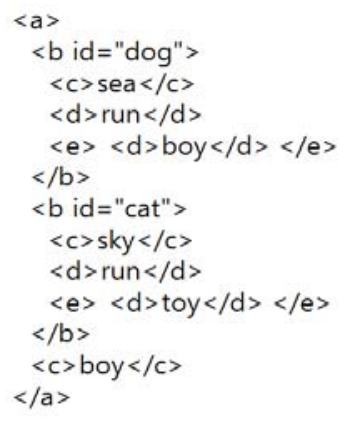

(a) XML Data

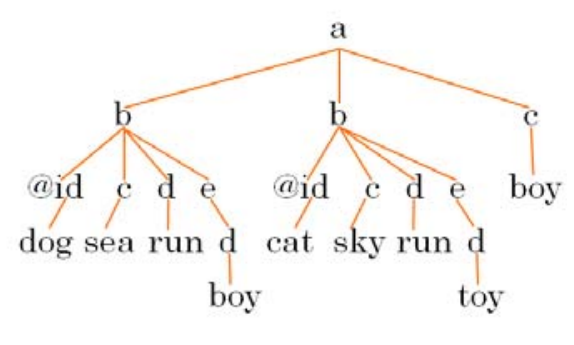

(b) XML Tree

Fig. 1. Sample XML Data and XML Tree

In storing an XML tree, it is shredded into XML nodes each of which is separately stored. The stored items associated with each node include its element tag name or attribute name (prefixed with @) or text/attribute value depending on its node type, its (begin:end) range, and its level if the range numbering scheme of [1] is employed. ${ }^{1}$ Often these nodes are indexed on the element tag/attribute name, on the text/attribute value, and on the "begin" value which is the unique identifier of each node. The former two types of indices are called name index and value index, respectively [4].

Put in a nutshell, the current state-of-the-art technique of storing XML data is node-based. The unit of storage is an XML node. In this paper, we investigate a generalization of such a

\footnotetext{
${ }^{1}$ Throughout this paper, we assume that the range numbering scheme of [1] is employed as the XML node labeling scheme. That choice is just for exposition of the concept. In fact, the technique proposed in this paper is independent of the XML node labeling scheme employed. Any scheme in the literature including the immutable ones for the dynamic XML documents can be employed for the technique proposed in this paper.
} 
technique. A subtree of the XML tree is considered as the unit of XML storage. For example, Fig. 3 shows a fragmentation of the XML tree of Fig. 1-(b) into subtrees each of which is marked by a triangle. Each subtree as a whole is stored as a unit of storage. At one extreme of the subtree-based XML storage, the entire XML tree could be stored as a whole without being shredded. At the other extreme, every XML node could be separately stored as in the conventional node-based technique. In this extreme, each node $n$ constitutes a subtree which consists of only $n$. In between, a subtree can consist of one or more nodes. Such a generalization is desirable for the following reasons:

1. A semantic unit of XML data matches the storage unit. For example, an employee element which includes employee ID, first name, last name, department, e-mail, and so on as subelements or attributes can be stored as a whole without being shredded. The whole XML subtree can be retrieved, transmitted and so on without re-constructuring the constituent XML nodes. Handling and exchanging XML data in subtrees is particularly essential in the applications like web service [5].

2. More efficient mapping of XML data into other data models such as relational or object-relational is possible. This problem received much attention [6][7] because XML data, in its semistructured nature, usually contains irregular portions, which cannot be efficiently mapped to relational or object-relational schema. Such overflow data can be stored as a subtree.

3. In the conventional node-based storage, there are quite many stored nodes produced for a large volume of XML data. The space required to store every node separately with its label could be large. More compact storage is possible with the subtree-based storage. The shredding of XML data into nodes is for efficient query processing. As for cold data against which queries are infrequent, it could be stored as a subtree, saving space.

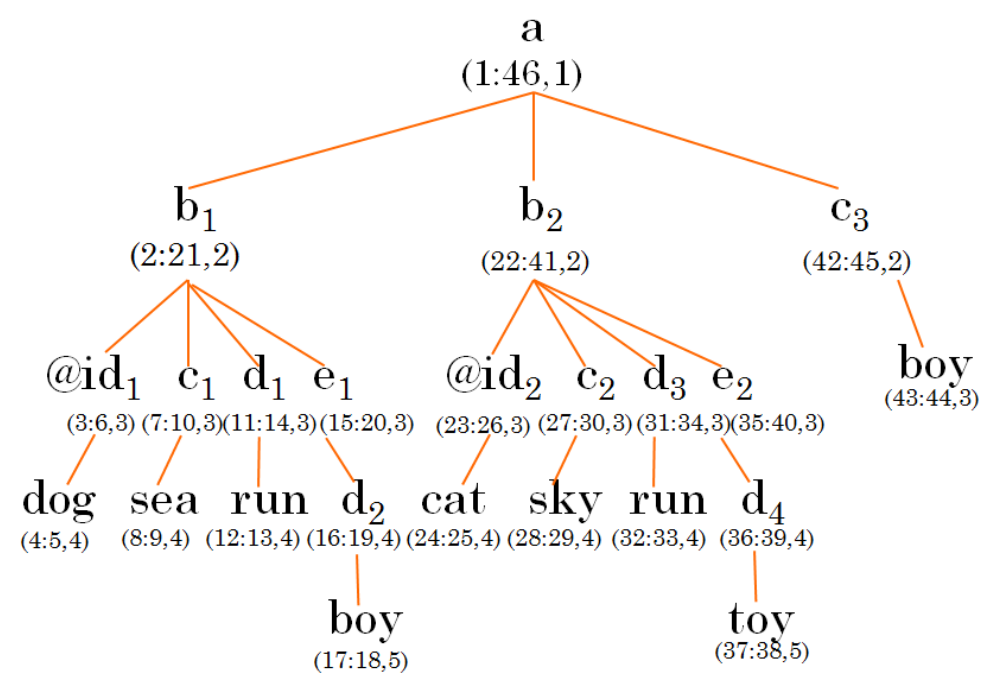

Fig. 2. XML Tree with Range Numbering

Despite these advantages, a major problem with subtree-based XML storage is inefficiency in query processing. Inefficiency arises when the XML nodes within a subtree need to be accessed. The subtree needs to be parsed on the fly. For example, in processing an XPath expressione, /a/b[@id='dog']//d, against the XML document of Fig. 1-(a) which is stored in subtrees as in Fig. 3, it is necessary to parse the subtree rooted at $b_{1}$ to access the attribute @id 
which resides inside the subtree. More subtrees need to be parsed in processing $e$. If the stored subtrees are eventually to be parsed for query processing, the afore-mentioned advantages are irrelevant or compromised.

In this paper, we propose a solution whereby no parsing of the stored subtree is needed at all in XPath processing unless the nodes of the final query result (i.e., node set) are to be retrieved from inside the subtrees. Such a solution is worth reporting because with it the subtree-based XML storage which has not been considered before in the literature would become a viable generalization of the conventional node-based XML storage.

The rest of this paper is organized as follows: In Section 2, we describe the subtree-based XML storage with the XPFS tree, which is the XML path and fragmentation schema needed in our proposed solution. In Section 3, we describe the conventional node-based XPath processing. In section 4, we present our technique of XPath processing where not any of the involved subtrees are parsed unless they contain the nodes of the final query result. We also prove that our technique guarantees the correctness of query processing. In Section 5, we report the implementation and experimental results, showing that the overhead of our solution is acceptable. In Section 6, we present related work. Conclusions and future work will be given in Section 7.

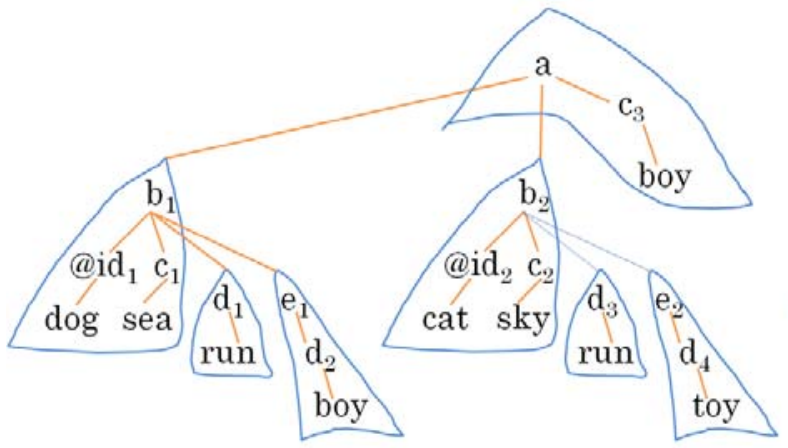

Fig. 3. Fragmentation of XML Tree into Subtrees

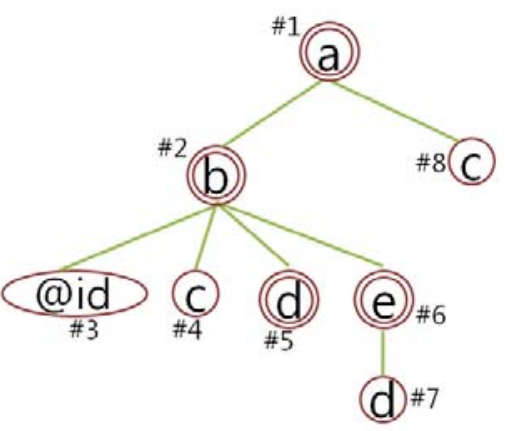

Fig. 4. XPFS Tree

\section{Subtree-based XML Storage with XPFS Tree}

To store XML data in subtrees and process XPath queries without parsing the subtrees involved, we need an XML path schema and an XML fragmentation schema. The former stores every path type, a sequence of element tag/attribute names, appearing in an XML document $D$ with their identifiers (i.e., path $I D$ ). They are identified while $D$ is parsed for its nodes to be labeled. The path schema can be represented as a tree as shown in Fig. 4, which is derived for the XML tree of Fig. 1-(b). The number (prefixed with \#) beside each node $N$ is the path ID of $N$, which is the path ID assigned to the path type from the root to $N$. For example, in Fig. 4, the path type $/ a / b / c$ is assigned \#4 as its path ID.

As for the fragmentation schema, it specifies how the original XML tree is fragmented into subtrees. The fragmentation schema can be incorporated into the path schema, resulting in an $X M L$ path and fragmentation schema(XPFS) tree as shown in Fig. 4. There are two types of nodes in an XPFS tree. One is marked with a circle, and the other with a double circle. A double circle node denotes the root of a subtree to be stored while a single circle one denotes a node that belongs to a subtree $t$ as a descendant of the root of $t$. In other words, given a double circle node $N, N$ and all its single circle descendants constitute a subtree to be stored. For example, the fragmentation shown in Fig. 3 conforms to the XPFS tree of Fig. 4. The nodes ' $a$ ', 
' $b$ ', ' $d$ ', and ' $e$ ' are in double circle in Fig. 4, and each of their instances is the root of the subtree marked by a triangle in Fig. 3.

For an XML document $D$ and its XPFS tree, $D$ is shredded into XML subtrees. Each subtree $t$ with $r$ as its root node is stored separately as a storage unit. The stored items associated with $t$ include the element tag/attribute name of $r$, the (begin:end) range and the level of $r$, path ID of $r$, and $t$ itself as a plain text not in any parsed format. Though $t$ is not parsed and thus not accessed during XPath processing in our technique, we leave a special symbol wherever a subtree is fragmented out of $t$. For example, Fig. 5 shows the subtree-based storage of the XML tree of Fig. 1-(b), which is fragmented as in Fig. 3. Seven subtrees are stored (subtree no. (1) through (7) in Fig. 5. The no.column is not stored. It is just for reference.) The subtrees (1), (2), and (5) contain the symbol "\$" as a marker for a subtree.

\begin{tabular}{|c|c|c|c|c|c|}
\hline no. & name & pathid & begin & end & value \\
\hline (1) & a & $\# 1$ & 1 & 46 & $<$ a $>\$ \$<c>$ boy $</$ c $></ a>$ \\
\hline (2) & b & \#2 & 2 & 21 & $<$ b id $="$ dog" $><$ c $>$ sea $</$ c $>\$ \$</$ b $>$ \\
\hline (3) & d & \#5 & 11 & 14 & $<$ d $>$ run $</$ d $>$ \\
\hline (4) & e & \#6 & 15 & 20 & $\langle$ e $\rangle\langle$ d $>$ boy $</$ d $\rangle\langle/$ e $\rangle$ \\
\hline (5) & b & $\# 2$ & 22 & 41 & $\langle$ b id $=$ "cat" $>\langle$ c $>$ sky $</$ c $>\$ \$</$ b $>$ \\
\hline (6) & d & $\# 5$ & 31 & 34 & $<$ d $>$ run $</$ d $>$ \\
\hline (7) & e & \#6 & 35 & 40 & $\langle$ e $\rangle\langle$ d $>$ toy $\langle/$ d $\rangle\langle/$ e $\rangle$ \\
\hline
\end{tabular}

Fig. 5. Subtree-based XML Storage

For comparison, Fig. 6 shows the conventional node-based storage of the same XML tree of Fig. 1-(b), where a total of 23 nodes are stored. (The no.column is not stored. It is for reference. As for the pathid column, let us ignore it for the moment. It will be mentioned later.) Comparing Fig. 5 with Fig. 6, we note that storing a subtree $t$ can be regarded as equal to storing only its root node $r$ except that path ID of $r$ and $t$ itself are stored as well. In XPath processing, $r$ represents the whole subtree $t$ without $t$ being parsed on the fly. ${ }^{2}$

In this paper, we assume that the XML documents conform to some DTD (Document Type Definition). The only rule we enforce in fragmenting an XML tree into subtrees is that the repeating or optional elements, which are specified with +, *, or ? in the DTD, are to be the roots of different subtrees. We call this rule basic XML fragmentation rule. In Fig. 3, for example, $b_{1}$ and $b_{2}$ are the repeating elements because they have the same parent ' $a$ ', and thus, the same path type $/ a / b$. Under the basic XML fragmentation rule, each of them is the root of a subtree as shown. As for the optional elements, if the XML nodes whose tag name is ' $d$ ' at the path type $/ a / b / d$ are optional, each of their instances, $d_{1}$ and $d_{3}$, is the root of a subtree as shown. The basic XML fragmentation rule plays a key role in subtree-based XPath processing as shall be shown in Section 4.

There are several related issues that are beyond the scope of this paper. For example, which nodes of the XPFS tree are marked with a double circle in coming up with an XPFS tree is important. So is updatability for dynamic XML documents. These and other issues are discussed in Section 7. In the remainder of this paper, we focus on subtree-based XPath processing where no subtree is parsed on the fly.

\footnotetext{
${ }^{2}$ As shall be shown in Section 4, as far as our subtree-based XPath processing is concerned, the element tag/attribute name and the level need not be stored because they are not refered to in XPath processing. The stored subtree $t$ itself is not accessed either unless $t$ contains the nodes of the final query result. Here, we include them all just to compare with the node-based storage.
} 


\begin{tabular}{|c|c|c|c|c|c|}
\hline no. & name & pathid & begin & end & level \\
\hline$(1)$ & a & $\# 1$ & 1 & 46 & 1 \\
\hline$(2)$ & b & $\# 2$ & 2 & 21 & 2 \\
\hline$(3)$ & @id & $\# 3$ & 3 & 6 & 3 \\
\hline$(4)$ & dog & - & 4 & 5 & 4 \\
\hline$(5)$ & c & $\# 4$ & 7 & 10 & 3 \\
\hline$(6)$ & sea & - & 8 & 9 & 4 \\
\hline$(7)$ & d & $\# 5$ & 11 & 14 & 3 \\
\hline$(8)$ & run & - & 12 & 13 & 4 \\
\hline$(9)$ & e & $\# 6$ & 15 & 20 & 3 \\
\hline$(10)$ & d & $\# 7$ & 16 & 19 & 4 \\
\hline$(11)$ & boy & - & 17 & 18 & 5 \\
\hline$(12)$ & b & $\# 2$ & 22 & 41 & 2 \\
\hline$(13)$ & $@ i d$ & $\# 3$ & 23 & 26 & 3 \\
\hline$(14)$ & cat & - & 24 & 25 & 4 \\
\hline$(15)$ & c & $\# 4$ & 27 & 30 & 3 \\
\hline$(16)$ & sky & - & 28 & 29 & 4 \\
\hline$(17)$ & d & $\# 5$ & 31 & 34 & 3 \\
\hline$(18)$ & run & - & 32 & 33 & 4 \\
\hline$(19)$ & e & $\# 6$ & 35 & 40 & 3 \\
\hline$(20)$ & d & $\# 7$ & 36 & 39 & 4 \\
\hline$(21)$ & toy & - & 37 & 38 & 5 \\
\hline$(22)$ & c & $\# 8$ & 42 & 45 & 2 \\
\hline$(23)$ & boy & - & 43 & 44 & 3 \\
\hline (2) & a & & & \\
\hline
\end{tabular}

Fig. 6. Node-based XML Storage

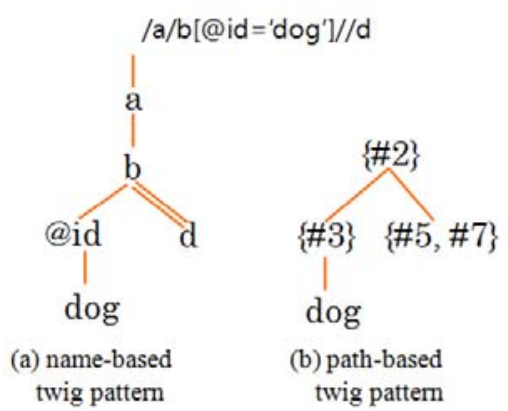

Fig. 7. Two Types of Twig Patterns

\section{Node-based XPath Processing}

In the literature, an XPath expression $e$ is represented as a tree $T(e)$ called twig pattern [1][2] [3]. Fig. 7-(a) shows an example for an XPath expression: /a/b[@id='dog']//d. A twig node of $T(e)$ is labeled with an element tag/attribute name or a text/attribute value. An edge between a pair of parent-child twig nodes is denoted either by a line or by a double line. The former represents the child axis (/) and the latter the self-or-descendant axis (//) in $e$. There is a special edge leading to the root of $T(e)$ : a line or a double line. If $e$ starts with a /, it is a line. If $e$ starts with a //, it is a double line.

With the node-based XML storage, the well-known node-based XPath processing scheme proceeds as follows [2]:

1. For each twig node, a list of corresponding XML nodes is retrieved as an input to structural join.

2. These XML node lists are scanned while node-based structural join is conducted to produce a node set as the final query result.

We note that the above popular formulation of an XPath expression $e$ as a twig pattern tree $T(e)$ and the processing of $e$ with $T(e)$ is name-based in the following sense:

1. Each twig node is labeled with an element tag/attribute name or a text/attribute value. (The text/attribute value can also be regarded as a name.)

2. For each twig node $N$, the XML node list for structural join is retrieved through name matching by which we mean that the element/attribute/text nodes whose name/value is equal to the name/value of $N$ are retrieved no matter which path they exist at.

Since we have the XPFS tree, for an XPath expression $e$, its name-based twig pattern $T_{\text {name }}(e)$ can be transformed into a path-based twig pattern $T_{\text {path }}(e)$ where each twig node is associated 
either with $a$ set of path IDs or with a text/attribute value. For example, the name-based twig pattern in Fig. 7-(a) is transformed into a path-based one as shown in Fig. 7-(b). For an XPath expression $e, T_{p a t h}(e)$ is obtained from $T_{\text {name }}(e)$ by mapping each liner path from the root to node $N$ in $T_{\text {name }}(e)$ into the set of corresponding path IDs where $N$ is either a branching node, a non-text leaf node, or the parent of a text/attribute value. For example, in $T_{\text {name }}(e)$ of Fig. 7-(a), there are three afore-mentioned linear paths: /a/b, /a/b/@id, and /a/b//d (' $b$ ' is a branching node, ' $d$ ' is a non-text leaf node, and '@id' is the parent of an attribute value.) Looking up the XPFS tree of Fig. 4, the path /a/b is mapped into $\{\# 2\}, / a / b / @ i d$ into $\{\# 3\}$, and $/ a / b / / d$ into $\{\# 5, \# 7\}$. (/a/b//d is resolved into $/ a / b / d$ or $/ a / b / e / d$.) If the linear path does not contain a $/ /$, it corresponds to a unique path type and is mapped to a set of just one path ID. Otherwise, it may be resolved into multiple path types and is mapped to a set of one or more path IDs. As for a text/attribute value in $T_{\text {name }}(e)$, it remains intact.

To conduct XPath processing for a path-based twig pattern, a slight modification is needed to the node-based XML storage. For each element/attribute node $n$ in the XML tree, the path ID of $n$ is stored and indexed. Instead, the element tag/attribute name of $n$ need not be stored. (In Fig. 5 and in Fig. 6, the pathid column is stored instead of the name column.) With such a modified XML storage, the node-based XPath processing scheme for a path-based twig pattern proceeds in a similar way to that for a name-based one:

1. For each non-text twig node labeled with a set of path IDs, $S$, a list of corresponding XML nodes is retrieved as follows: For each path ID $I D_{i} \in S$, the set of all the XML nodes whose path ID is $I D_{i}$ is retrieved. Union of these node sets forms the list. For this, an index on path ID is desired. For a text twig node, its list is retrieved through the value index.

2. These XML node lists are scanned for structural join to produce a node set as the final query result.

Such name-based to path-based transformation was materialized in XRel [8]. Since XRel is an XML storing and XPath processing system built on top of an RDBMS, all the path types in XML documents were enumerated and stored in a table called PATH with their path IDs. In translating an XPath expression into an SQL statement, such a transformation occurs. In the remainder of this paper, we deal only with the XPath processing for the path-based twig pattern. The equivalence between XPath processing for a path-based twig pattern and that for its original name-based counterpart is referred to [8].

\section{Subtree-based XPath Processing}

\subsection{Overview}

With a subtree-based XML storage, the conventional node-based XPath processing seems impossible without parsing the stored subtrees involved in the query. To come up with the query result, for each twig node, a list of XML nodes needs to be retrieved for structural join. Since the relevant XML nodes may reside inside the stored subtrees, it seems inevitable to retrieve the subtrees and parse them on the fly. For a node $n$ inside a subtree $t$, actually there is no way to isolate and retrieve $n$ without parsing $t$. Note that $n$ was not stored or indexed separately on its own. Rather, what is stored is $t$ (more specifically, the root node of $t$ ) and what is possibly indexed is just the root node of $t$. The values of the text/attribute nodes inside $t$ cannot be indexed, either. (They simply cannot be accessed without parsing $t$.) Only the root node of $t$ with its (begin:end) range and its path ID can be accessed. Then, how could an XPath 
query be processed without the subtrees involved being parsed? In this section, we propose a solution.

In the node-based XPath processing, both the input and the output of structural join are XML node sets. Note that this is not the case for the subtree-based processing simply because the XML nodes may reside inside subtrees and could not be accessed. As such, the input and the output of structural join are subtrees, the unit of storage. More specifically, the differences between node-based and subtree-based XPath processing for a path-based twig pattern are as follows:

1. In the node-based processing, for each twig node, an XML node list $\left(n_{1}, \ldots, n_{j}\right)$ is retrieved where each $n_{i}$ is represented by the (begin:end) range of $n_{i}$. In the subtree-based processing, for each twig node, a list of <path ID, subtree> pairs $\left(<I D_{1}, t_{1}>, \ldots,<I D_{j}, t_{j}>\right)$ is retrieved. A pair $<I D_{i}, t_{i}>$ corresponds to $n_{i}$ such that $I D_{i}$ is the path ID of $n_{i}$ and $t_{i}$ is the subtree to which $n_{i}$ belongs. Since for a subtree $t$, its root node is to represent $t$ in XPath processing, what is really retrieved is a list of $<$ path ID, the root of a subtree $>$ pairs $\left.\left.\left(<I D_{1}, r_{1}\right\rangle, \ldots,<I D_{j}, r_{j}\right\rangle\right)$ where $r_{i}$ is the root node of $t_{i}$ and represented by its (begin:end) range. As mentioned in Section 2, the (begin:end) range

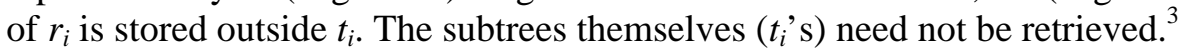

2. The final query result in the node-based processing is an XML node set $\left\{n_{1}, \ldots, n_{k}\right\}$ as defined in the XPath specification [9]. That should be the same in the subtree-based processing but here subtrees, not individual nodes, are dealt with. Thus, a set of $<$ path ID, subtree $>$ pairs, $\left.\left\{\left\langle I D_{1}, t_{1}\right\rangle, \ldots,<I D_{k}, t_{k}\right\rangle\right\}$ are returned first as a pre-result. To be exact, a set of $<$ path ID, the root of a subtree $>$ pairs $\left.\left.\left(<I D_{1}, r_{1}\right\rangle, \ldots,<I D_{k}, r_{k}\right\rangle\right)$ is returned where $r_{i}$ is the root of $t_{i}$. For the final result, each $t_{i}$ is retrieved from the "begin" value of $r_{i}$, and each $n_{i}$ whose path ID is $I D_{i}$ is retrieved from $t_{i}$. Only in this stage, parsing of $t_{i}$ is needed.

Our proposed subtree-based XPath processing scheme proceeds as follows: Given an XPath expression $e$,

STEP 1: its name-based twig pattern $T_{\text {name }}(e)$ is transformed into a path-based one $T_{p a t h}(e)$. STEP 2: For each twig node of $T_{\text {path }}(e)$, the input subtree list is retrieved from the subtree-based XML storage.

STEP 3: These subtree lists are scanned while subtree-based structural join is conducted to produce a set of subtrees as a pre-result.

STEP 4: The final result node set is obtained from the pre-result subtrees.

The above steps 1 and 4 are already described. The steps 2 and 3 are described below.

\subsection{Input of Subtree-based XPath Processing}

In STEP 2, to retrieve the input subtree list from the subtree-based XML storage, for each non-text twig node labeled with a set of path IDs $S=\left\{I D_{1}, \ldots, I D_{j}\right\}, S$ is first converted to a new set $S_{r}=\left\{\left\langle I D_{1}, R\left(I D_{1}\right)\right\rangle, \ldots .,\left\langle I D_{j}, R\left(I D_{j}\right)\right\rangle\right\}$ where the function $R$ called root function is defined as follows:

\section{Definition 4.1 [Root Function]}

For a node $N$ in an XPFS tree $T$, let $x$ be the path ID of the path type from the root of $T$ to $N$. Let $N_{r}$ be the root node (i.e., one in double circle) of the subtree to which $N$ belongs in $T$. Then,

${ }^{3}$ In the remainder of this paper, we use the followings interchangeably: a list of subtrees, a list of $<$ path ID, subtree> pairs, and a list of < path ID, the root of a subtree> pairs. 
$R(x)=y$ where $y$ is the path ID of the path type from the root of $T$ to $N_{r}$.

\section{Example 4.1}

In Fig. 4, $R(\# 3)=\# 2$ because the node '@id' (path ID=\#3) is a child of ' $b$ ' (path ID=\#2) in double circle. $R(\# 7)=\# 6$ because the node ' $d$ ' at $/ a / b / e / d$ (path ID=\#7) is a child of ' $e$ ' (path $\mathrm{ID}=\# 6$ ) in double circle. Meanwhle, $R(\# 5)=\# 5$ and $R(\# 2)=\# 2$ because for the double circle node, the root function returns the path ID of itself.

The conversion of $S$ to $S_{r}$ is to retrieve the input subtree list for structural join instead of the XML node list, which cannot be retrieved without parsing the subtrees. For each $\left\langle I D_{i}, R\left(I D_{i}\right)>\right.$ in $S_{r}$, the set of all the subtrees the path ID of whose root is $R\left(I D_{i}\right)$ is retrieved. These subtrees are those that contain the target XML nodes whose path ID is $I D_{i}$. Union of these subtree sets forms the input list for the twig node labeled with $S_{r}$.

\begin{tabular}{|c|c|}
\hline <pathid, value $>$ & $\begin{array}{c}\text { node pointers } \\
\text { (no. column in } \\
\text { Fig. 6) }\end{array}$ \\
\hline$<\# 3$, dog $>$ & $\{(4)\}$ \\
\hline$<\# 4$, sea $>$ & $\{(6)\}$ \\
\hline$<\# 5$, run> & $\{(8),(18)\}$ \\
\hline$<\# 7$, boy $>$ & $\{(11)\}$ \\
\hline$<\# 3$, cat $>$ & $\{(14)\}$ \\
\hline$<\# 4$, sky> & $\{(16)\}$ \\
\hline$<\# 7$, toy> & $\{(21)\}$ \\
\hline$<\#$, boy> & $\{(23)\}$ \\
\hline
\end{tabular}

(a) index entries for node-based storage

\begin{tabular}{|c|c|}
\hline <pathid, value $>$ & $\begin{array}{c}\text { subtree pointers } \\
\text { (no. column in } \\
\text { Fig. 5) }\end{array}$ \\
\hline$<\# 3$, dog> & $\{(2)\}$ \\
\hline$<\# 4$, sea> & $\{(2)\}$ \\
\hline$<\#$, run> & $\{(3),(6)\}$ \\
\hline$<\#$, boy> & $\{(4)\}$ \\
\hline$<\# 3$, cat $>$ & $\{(5)\}$ \\
\hline$<\# 4$, sky> & $\{(5)\}$ \\
\hline$<\#$ 7, toy> & $\{(7)\}$ \\
\hline$<\# 8$, boy> & $\{(1)\}$ \\
\hline
\end{tabular}

(b) index entries for subtree-based storage

Fig. 8. Composite Value Index

As for the text twig node with a value $v$, all the text nodes whose value is $v$ need to be retrieved for structural join. They may reside inside the stored subtrees. We handle this problem through a modified value index as follows: An index entry of the conventional value index for a node-based XML storage is of the form $(v, P)$ where $v$ is a value and $P$ is a set of pointers to the text nodes whose value is $v$. Such typical indexing is for the name-based twig pattern, and the pointers in $P$ are supposed to point to all the text nodes whose value is $v$ no matter which path they exist at. We first modify it so that it well fits the path-based twig pattern. A composite value index whose entry is of the form $(\langle I, v\rangle, P)$ is used where

1. I is the path ID of the path type from the root of the XML document to the parent node of the text node $N_{t}$ whose value is $v$.

2. $P$ is a set of pointers to the instances of such $N_{t}$.

With a subtree-based storage, however, a text node inside a subtree cannot be pointed to directly. Thus, $P$ needs further modification: $P$ is a set of pointers $\left\{p_{1}, \ldots, p_{q}\right\}$ where each $p_{k}$ points to the root of the subtree to which the instances of $N_{t}$ belongs. 


\section{Example 4.2}

Fig. 8-(a) shows the index entries for the XML tree of Fig. 1-(b) with the node-based XML storage of Fig. 6. The pointers are represented by the reference number in no.column in Fig. 6. Fig. 8-(b) shows the index entries for the XML tree of Fig. 1-(b) with the subtree-based XML storage of Fig. 5. The pointers are represented by the reference number in no.column in Fig. $\mathbf{5}$.

For a text twig node $N_{t}$ labeled with a value $v$ whose parent twig node is $N_{p}$ labeled with a set of path IDs $S=\left\{I D_{1}, \ldots, I D_{j}\right\}$ (or with its converted set $S_{r}=\left\{\left\langle I D_{1}, R\left(I D_{1}\right)>, \ldots .,<I D_{j}\right.\right.$, $R\left(I D_{j}\right)>$ \}), the input subtree list for $N_{t}$ is the union of $\left\langle I D_{i}, S T\left(p_{k}\right)>, i=1, \ldots, j\right.$ and $k=1, \ldots, q$ where $p_{k} \in P_{i}$ such that $P_{i}$ is the set of pointers of the value index entry $\left(\left\langle I D_{i}, v\right\rangle, P_{i}\right)$ and $S T\left(p_{k}\right)$ denotes the subtree pointed to by $p_{k}$.

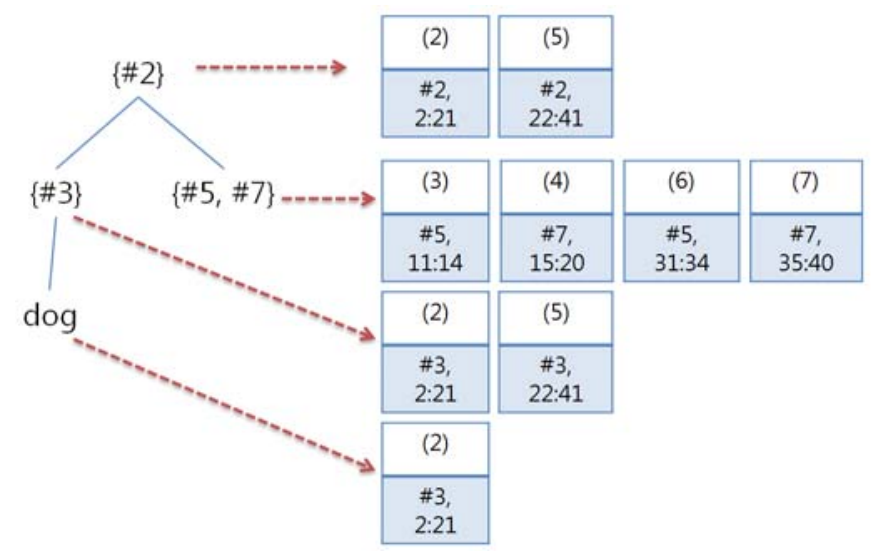

Fig. 9. Input Subtree Lists

\section{Example 4.3}

Fig. 9 shows the input subtree lists for the path-based twig pattern of Fig. 7-(b) retrieved from the subtree-based XML storage of Fig. 5. For the twig nodes labeled with $\{\# 2\}$, $\{\# 3\}$, and $\{\# 5, \# 7\}$, two, two, and four subtrees are retrieved, respectively. Each box corresponds to a subtree $t$ that contains the target XML node $n$, denoted by $<(i) j, k: l>$ where (i) is the reference number of $t$ (in no.column of Fig. 5), $j$ is the path ID of $n$, and $k: l$ is the (begin:end) range of the root of $t$. The first subtree denoted by $<(2) \# 3,2: 21>$, for the twig node labeled with $\{\# 3\}$, for example, corresponds to the subtree rooted at ' $b_{1}$ ' in Fig. 3, and its target XML node is '@id,'.

Now STEP 2 is completed, and every twig node is provided with its input subtree list. We are ready for STEP 3 described in the next subsection.

\subsection{Subtree-based Structural Join}

A structural join between two lists of XML nodes is well-known [1][2][4]. The basis of node-based structural join is that given two nodes $n_{1}$ and $n_{2}$ with their (begin:end) ranges, their structural relationship (or containment relationship) can be determined by comparing their ranges. In the case of subtree-based XML storage, $n_{1}$ and $n_{2}$ may reside inside some subtrees and their ranges are not given. It could also be that $n_{1}$ and $n_{2}$ may reside in the same subtree. How could the structural relationship between $n_{1}$ and $n_{2}$ be determined without parsing the subtrees? In this subsection, we show that such subtree-based structural join can be correctly conducted. 
A path type in an XML document $D$ can be defined as a sequence of element tag/attribute names. It corresponds to a path in the XPFS tree of $D$. For two path types, one can be a prefix of the other. For example, in the XPFS tree of Fig. 4, the path type /a/b is a prefix of the path types $/ a / b / c, / a / b / e / d$, and so on. A path type is also a prefix of itself. For an XML node $n$ in an XML document $D$, let $P(n)$ denote the path type of the path from the root of $D$ to $n$. For example, in the XML tree of Fig. 2, $P\left(b_{1}\right)=/ a / b, P\left(c_{1}\right)=/ a / b / c$, and $P\left(c_{3}\right)=/ a / c$. (We note that $P\left(b_{1}\right)$ is a prefix of $P\left(c_{1}\right)$ but $P\left(c_{3}\right)$ is not a prefix of $P\left(c_{1}\right)$.) For an XML node $n$, let range(n) denote the (begin:end) range of $n$, and for a stored subtree $t$ whose root node is $r$, let range(t) denote the range( $r$ ). Then, with a subtree-based XML storage of an XML document $D$ derived by the XPFS tree with the basic XML fragmentation rule as described in Section 2, we have the following Theorem.

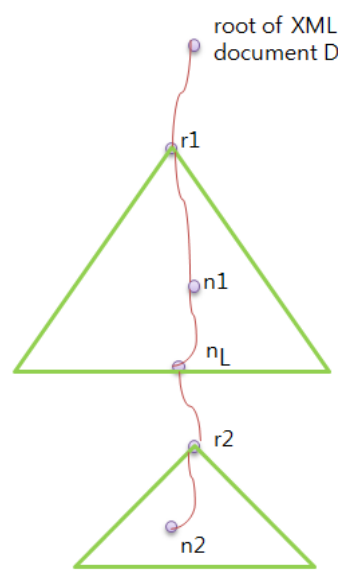

(a)

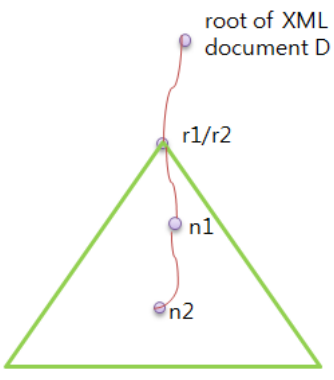

(b)

Fig. 10. Two Cases in Theorem 4.1

\section{Theorem 4.1}

For two XML nodes $n_{1}$ and $n_{2}$ which respectively belong to subtrees $t_{1}$ and $t_{2}$ in an XML document $D$ stored in a subtree-based storage derived by the XPFS tree of $D, n_{1}$ is an ancestor of $n_{2}$ iff the following two conditions hold:

1. range $\left(t_{1}\right)$ properly contains or is equal to $\operatorname{range}\left(t_{2}\right)$.

2. $P\left(n_{1}\right)$ is a prefix of $P\left(n_{2}\right)$

\section{Proof}

(if) Suppose the above two conditions hold. Then, we show that $n_{1}$ is an ancestor of $n_{2}$. Let $r_{1}$ and $r_{2}$ be the root node of $t_{1}$ and $t_{2}$, respectively (cf. Fig. 10). In case that range $\left(t_{1}\right)$ properly contains range $\left(t_{2}\right)$, there is a leaf node $n_{L}$ in $t_{1}$ which is the parent of $r_{2}$. In case that range $\left(t_{1}\right)$ is equal to range $\left(t_{2}\right), t_{1}$ and $t_{2}$ are the same subtree. That is, $r_{1}$ and $r_{2}$ are the same, and $n_{1}$ and $n_{2}$ are in the same subtree. Due to the basic XML fragmentation rule, for a path type $P$, there could be at most one instance of an XML node in a subtree whose path type is $P$. As such, there is not a node $m$ in $t_{1}$ such that $P(m)=P\left(n_{1}\right)$. Similarly, there is not a node $m$ in $t_{2}$ such that $P(n)=P\left(n_{2}\right)$. In other words, $n_{1}$ is the unique node in $t_{1}$ whose path type is $P\left(n_{1}\right)$, and $n_{2}$ is the unique node in $t_{2}$ whose path type is $P\left(n_{2}\right)$. As such, $n_{1}$ and $n_{2}$ must be on the same path $p$ because $P\left(n_{1}\right)$ is a prefix of $P\left(n_{2}\right)$ where $p$ is as follows: In case that range $\left(t_{1}\right)$ properly contains range $\left(t_{2}\right), p$ is the path: the root of $D-r_{1}-n_{1}-n_{L}-r_{2}-n_{2}$ (cf. Fig. 10-(a)). In case that range $\left(t_{1}\right)$ is 
equal to range $\left(t_{2}\right), p$ is the path: the root of $D-r_{1}\left(r_{2}\right)-n_{1}-n_{2}$ (cf. Fig. 10-(b)). In all, $n_{1}$ is an ancestor of $n_{2}$.

(only if) Suppose $n_{1}$ is an ancestor of $n_{2}$. Then, we show that the above two conditions hold. $t_{1}$ and $t_{2}$ are either the same subtree or different ones. In case that $t_{1}$ and $t_{2}$ are the same, range $\left(t_{1}\right)$ is equal to range $\left(t_{2}\right)$. In case that $t_{1}$ and $t_{2}$ are different, let $r_{1}$ and $r_{2}$ be the root node of $t_{1}$ and $t_{2}$, respectively. Since $n_{1}$ is an ancestor of $n_{2}$, they are on the same path: the root of $D-r_{1}-n_{1}-n_{L}-r_{2}-n_{2}$ where $n_{L}$ is a leaf node in $t_{1}$ which is the parent of $r_{2}$ (cf. Fig. 10-(a)). Since range $\left(t_{1}\right)$ properly contains or is equal to range $\left(n_{L}\right)$, range $\left(n_{L}\right)$ properly contains $\operatorname{range}\left(r_{2}\right)$, and range $\left(r_{2}\right)$ is range $\left(t_{2}\right)$, range $\left(t_{1}\right)$ properly contains range $\left(t_{2}\right)$. Therefore, the first condition holds. As for the second condition, $P\left(n_{1}\right)$ must be a prefix of $P\left(n_{2}\right)$ because $n_{1}$ is an ancestor of $n_{2}$.

Theorem 4.1 is the basis of the subtree-based structural join. As described in the previous subsection, for each twig node in the path-based twig pattern for an XPath expression, the list of $<$ path ID, subtree $>$ pairs is retrieved for structural join. Each pair $\left\langle I D_{i}, t_{i}>\right.$ corresponds to an XML node $n_{i}$ that belongs to the subtree $t_{i}$. The path type of $n_{i}$ can be obtained by looking up the XPFS tree with its path ID $I D_{i}$. The (begin:end) range of $t_{i}$ is a priori given because what represents $t_{i}$ in the pair $<I D_{i}, t_{i}>$ is the (begin:end) range of the root node of $t_{i}$. As such, the structural relationship between two XML nodes $n_{1}$ and $n_{2}$ in an XML document stored in subtrees can be determined given two pairs $\left\langle I D_{1}, t_{1}\right\rangle$ and $\left\langle I D_{2}, t_{2}\right\rangle$ which respectively correspond to $n_{1}$ and $n_{2}$ without parsing $t_{1}$ or $t_{2}$.

Given a path-based twig pattern for an XPath expression $e$, each of its twig nodes is first provided with the input list of <path ID, subtree> pairs. Then, a series of subtree-based structural joins between two twig nodes $N_{p}$ and $N_{c}$ such that $N_{p}$ is the parent of $N_{c}$ is conducted. That obtains the pre-result of $e$, which is a set of <path ID, subtree> pairs. Each pair corresponds to an XML node in the node set that would be produced as the final result of $e$.

\subsection{Correctness of Subtree-based XPath Processing}

The correctness of the processing of an XPath expression against a subtree-based XML storage can be defined as follows:

\section{Definition 4.2}

In an XML document $D$, a $<$ path ID, subtree $>$ pair $<$ I, $t>$ where $t$ is a subtree of $D$ is said to uniquely cover an XML node $n$ of $D$ iff

1. $n$ exists in $t$ and

2. $n$ is the only XML node in $t$ whose path ID is $I$.

\section{Definition 4.3}

In an XML document $D$, let $S_{T}$ and $S_{n}$ be a set of <path ID, subtree> pairs and a set of XML nodes, respectively. $S_{T}$ is said to uniquely cover $S_{n}$ iff the elements of $S_{T}$ are in one-to-one correspondence with those of $S_{n}$ such that for each corresponding pair $\left(\left\langle I D_{i}, t_{i}\right\rangle, n_{i}\right)$ where $\left\langle I D_{i}\right.$, $t_{i}>\in S_{T}$ and $n_{i} \in S_{n},<I D_{i}, t_{i}>$ uniquely covers $n_{i}$.

\section{Definition 4.4}

For an XML document $D$, its XPFS tree, and an XPath expression $e$, let $R_{n}$ be the XML node set which is the final result of $e$ retrieved against the node-based storage of $D$ and let $P R_{T}$ be the set of <path ID, subtree> pairs which is the pre-result of $e$ retrieved against a subtree-based storage of $D$ derived by the XPFS tree. $P R_{T}$ is correct iff $P R_{T}$ uniquely covers $R_{n}$. 
The correctness of $P R_{T}$ implies that if $P R_{T}$ is correct, it is guaranteed to correctly get $R_{n}$ out of $P R_{T}$ in STEP 4 of the subtree-based XPath processing.

\section{Example 4.4}

For XPath expression $e, / a / b[@ i d=' d o g$ '] $/ / d$, the result of $e$ against the XML tree of Fig. 2 obtained by the conventional node-based XPath processing is the node set $R_{n}=\left\{d_{1}, d_{2}\right\}$. The pre-result of $e$ against the subtree-based XML storage shown in Fig. 3 obtained by the subtree-based XPath processing is the set $P R_{T}=\left\{<\right.$ path ID of $d_{1}$, subtree rooted at $d_{1}>$, <path ID of $d_{2}$, subtree rooted at $\left.e_{1}>\right\}$. We note that $P R_{T}$ and $R_{n}$ are in one-to-one correspondence such that <path ID of $d_{1}$, subtree rooted at $d_{1}>$ uniquely covers $d_{1}$ and that <path ID of $d_{2}$, subtree rooted at $e_{1}>$ uniquely covers $d_{2}$. Thus, $P R_{T}$ uniquely covers $R_{n}$, and $P R_{T}$ is correct.

\section{Theorem 4.2}

For an XML document $D$, its XPFS tree, and an XPath expression $e$, the pre-result of $e$ for the path-based twig pattern of $e$ against a subtree-based XML storage of $D$ derived by the XPFS tree, obtained by STEP 2 and then by STEP 3 of the previous subsections without parsing the subtrees involved, is correct.

\section{Proof}

Let $R_{n}(e)$ and $P R_{T}(e)$ be the final result of $e$ obtained against the node-based storage of $D$ and the pre-result of $e$ obtained against the subtree-based storage of $D$. Let $T(e)$ be the path-based twig pattern of $e$. For each twig node of $T(e)$, let $L_{n}$ and $L_{T}$ be the input XML node list retrieved from the node-based storage of $D$ and the input list of $<$ path ID, subtree> pairs retrieved from the subtree-based storage of $D$, respectively. Because of the basic XML fragmentation rule described in Section 2 and the tasks of STEP 2, we see that $L_{T}$ uniquely covers $L_{n}$. As such, we see that due to Theorem 4.1, $P R_{T}(e)$ obtained in STEP 3 by a series of structural joins uniquely covers $R_{n}(e)$. Thus, by Definition $4.4, P R_{T}(e)$ is correct.

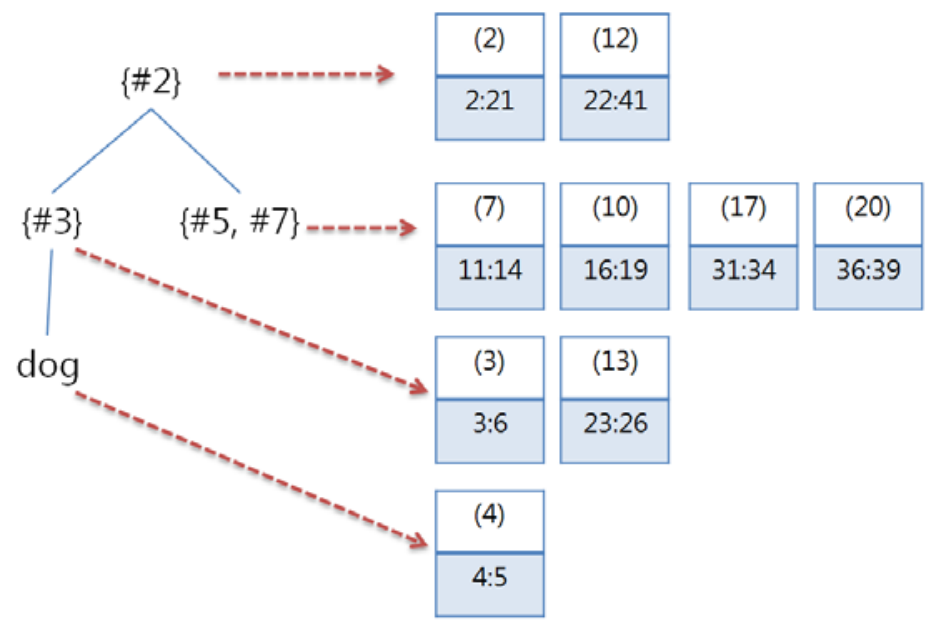

Fig. 11. Input XML Node Lists

\section{Example 4.5}

Let us consider an XPath expression e, /a/b[@id= 'dog']//d. Fig. 11 shows the path-based twig pattern for $e$ with its input XML node lists retrieved from the node-based XML storage of Fig. 6. (Note that Fig. 11 is the node-based version of Fig. 9. The latter shows the same twig pattern 
with its input lists of <path ID, subtree> pairs retrieved from the subtree-based XML storage of Fig. 5). For the twig nodes labeled with $\{\# 2\},\{\# 3\}$, and $\{\# 5, \# 7\}$, two, two, and four XML nodes are retrieved, respectively. Each box corresponds to an XML node $n$ denoted by $<(i)$ $j: k>$ where (i) is the reference number of $n$ (in no.column of Fig. 6), and $j: k$ is the (begin:end) range of $n$.

Let us compute $R_{n}(e)$ in Fig. 11 by a series of structural joins. Joining two twig nodes $\{\# 3\}$ and 'dog' (i.e., joining $\{(3),(13)\}$ vs. $\{(4)\})$ produces the XML node (3) for the twig node $\{\# 3\}$ as an intermediate result. Joining twig nodes $\{\# 2\}$ and $\{\# 3\}$ produces the XML node (2) for the twig node $\{\# 2\}$. Finally, joining twig nodes $\{\# 2\}$ and $\{\# 5, \# 7\}$ produces the final result node set $R_{n}(e)=\{(7),(10)\}$, which corresponds to $d_{1}$ and $d_{2}$ in Fig. 2 .

Now let us compute $P R_{T}(e)$ in Fig. 9. The whole process is the same as above except that the path IDs are checked as well to see if one path type is a prefix of the other. Joining two twig nodes $\{\# 3\}$ and 'dog' (i.e., joining $\{<\# 3$, subtree (2)>, $<\# 3$, subtree (5) $>\}$ vs. $\{<\# 3$, subtree $(2)>\}$ ) produces the $<\# 3$, subtree (2) $>$ for the twig node $\{\# 3\}$ as an intermediate result. All the path IDs here are \#3. Since they are the same, the prefix condition is satisfied. Joining twig nodes $\{\# 2\}$ and $\{\# 3\}$ produces the $<\# 2$, subtree (2)> for the twig node $\{\# 2\}$. Here, the path IDs involved are \#2 and \#3. Looking up the XPFS tree of Fig. 4, the prefix condition also holds. Finally, joining twig nodes $\{\# 2\}$ and $\{\# 5, \# 7\}$ produces the pre-result subtree set $P R_{T}(e)=\{<\# 5$, subtree (3)>, <\#7, subtree (4)>\}, which corresponds to $\left\{<\right.$ path ID of $d_{1}$, subtree rooted at $d_{1}>$, $<$ path ID of $d_{2}$, subtree rooted at $\left.e_{1}>\right\}$ in Fig. 3. As examined in Example 3.4, $P R_{T}(e)$ uniquely covers $R_{n}(e)$. Thus, $P R_{T}(e)$ is correct.

\section{Implementation and Experiments}

\subsection{Implementation}

We have implemented in Java two XML storing and XPath processing systems as described in previous sections. One is with the node-based XML storage and XPath processing (System/ $\mathrm{N}$ ), and the other is with the subtree-based XML storage and XPath processing $($ System/T). For ease of implementation, an RDBMS, Oracle 11g, was employed for storing and indexing in both systems. In System/N, each node is stored as a record of a table called NODE whereas in System/T, each subtree is stored as as a record of a table called SUBTREE.

The auction.xml generated in XMark [10] was used as the XML document. XPath expressions that appear in the XQuery FLWOR expressions in XMark were used to confirm the correctness of the proposed subtree-based XPath processing. They are listed in Table 1. The XPath processing time in System/T and System/ $\mathrm{N}$ was measured and compared. Also the space requirement in System/ $\mathrm{T}$ and System $/ \mathrm{N}$ was measured and compared. The implementation and experiments were carried out on a Windows XP server with Intel Core 2 Duo 6600 (2.40Ghz) CPU and 2 GB memory.

Table 1. XPath Expressions

\begin{tabular}{|c|c|}
\hline Query & XPath Expressions \\
\hline \hline Q1 & /site/people/person[homepage]/name \\
\hline Q2 & /site/closed_auctions/closed_auction/price \\
\hline Q3 & /site//item[description]/name \\
\hline Q4 & /site/regions/europe/item[@id]/name \\
\hline Q5 & /site/people/person[profile/interest/@category]/address/city \\
\hline
\end{tabular}




\subsection{XPath Processing Time}

For all the XPath expressions in Table 1, the correctness of the proposed subtree-based XPath processing was confirmed with auction.xml of size 1.16 MB. Though the goal of the technique proposed in this paper is not for improving query performance, we checked query performance of System/T in comparison with that of System/ $\mathrm{N}$ by carrying out two experiments. The two differ only in System/T. In the first experiment, for every query, the final result XML nodes are set to be the descendants of the root of their subtrees. In the second experiment, for every query, the final result XML nodes are set to be the root of their subtrees. The difference is the necessity of the parsing of the subtrees in the final stage of subtree-based XPath processing (i.e., STEP 4) in System/T: parsing is needed in the first experiment, whereas it is not in the second. Table 2 shows the time in System/N $\left(T_{N}\right)$ and in Syetem/T $\left(T_{T}\right)$ out of the first experiment and Table 3 out of the second. Each of the measured time is the average of 100 runs. As shown in Table 2, System/T took more time than System/ $\mathrm{N}$ because of the parsing overhead. However, as shown in Table 3, System/T outperforms System/N.

Table 2. Query Processing Time (ms) (result node: inside the subtree)

\begin{tabular}{|c|c|c|c|}
\hline Query & $\mathbf{T}_{\mathbf{N}}$ & $\mathbf{T}_{\mathbf{T}}$ & $\mathbf{T}_{\mathbf{T}} / \mathbf{T}_{\mathbf{N}}$ \\
\hline \hline Q1 & 65.90 & 97.61 & 1.48 \\
\hline Q2 & 46.56 & 80.08 & 1.72 \\
\hline Q3 & 154.71 & 261.92 & 1.69 \\
\hline Q4 & 48.13 & 88.84 & 1.85 \\
\hline Q5 & 51.52 & 59.04 & 1.15 \\
\hline
\end{tabular}

Table 3. Query Processing Time (ms) (result node: root of the subtree)

\begin{tabular}{|c|c|c|c|}
\hline Query & $\mathbf{T}_{\mathbf{N}}$ & $\mathbf{T}_{\mathbf{T}}$ & $\mathbf{T}_{\mathbf{T}} / \mathbf{T}_{\mathbf{N}}$ \\
\hline \hline Q1 & 66.37 & 19.45 & 0.29 \\
\hline Q2 & 49.68 & 15.17 & 0.31 \\
\hline Q3 & 154.62 & 19.96 & 0.13 \\
\hline Q4 & 48.26 & 16.57 & 0.34 \\
\hline Q5 & 51.52 & 22.22 & 0.43 \\
\hline
\end{tabular}

There are mainly two reasons for the superior query performance of System/T in the second experiment. First, as shall be described shortly, the size of the table NODE is much larger than that of SUBTREE. As such, the time to retrieve the input node lists for the twig nodes in System/ $\mathrm{N}$ took much longer than the time to retrieve the input subtree lists for the twig nodes in System/T. Secondly, the extra work done in the subtree-based XPath processing compared with the node-based counterpart is not burdensome, calling the root function in retrieving the input subtree lists and checking if one path type is a prefix of another in structural join.

Table 4. Space Overhead for XPFS Tree

\begin{tabular}{|c|c|c|c|}
\hline $\begin{array}{c}\text { Scaling } \\
\text { factor for } \\
\text { auction.xml }\end{array}$ & $\begin{array}{c}\text { Size of auction.xml } \\
\text { (KB) }\end{array}$ & $\begin{array}{c}\mathbf{S}_{\mathbf{X}} \\
\text { Size of } \mathbf{X P F S} \text { Tree } \\
\mathbf{( K B )}\end{array}$ & $\begin{array}{c}\mathbf{S}_{\mathbf{T}} \\
\left(\mathbf{S}_{\mathbf{T}} / \mathbf{S}_{\mathbf{X}}\right) \\
\mathbf{( \% )}\end{array}$ \\
\hline \hline 0.01 & 1,155 & 49.2 & 4.26 \\
\hline 0.02 & 2,330 & 51.6 & 2.21 \\
\hline 0.04 & 4,727 & 56.4 & 1.19 \\
\hline 0.06 & 6,984 & 57.9 & 0.83 \\
\hline 0.08 & 9,371 & 58.6 & 0.63 \\
\hline 0.1 & 11,597 & 59.1 & 0.51 \\
\hline 0.2 & 23,365 & 60.5 & 0.26 \\
\hline 0.4 & 46,395 & 60.5 & 0.13 \\
\hline 0.6 & 69,892 & 60.5 & 0.09 \\
\hline 0.8 & 92,975 & 60.5 & 0.07 \\
\hline 1 & 115,775 & 60.5 & 0.05 \\
\hline
\end{tabular}




\subsection{Space Requirement}

The subtree-based XML storage is much more compact than the node-based counterpart. In the first experiment mentioned above, for auction.xml of size 1.16 MB, there are 21,051 records stored in the table NODE, and 4,791 records in the table SUBTREE (Note that when the XML tree of Fig. 1-(b) is stored, there are 23 nodes in the node-based XML storage in Fig. 6 while there are just 7 subtrees in the subtree-based counterpart in Fig. 5. The space occupied by NODE and SUBTREE in the table space of Oracle is roughly 0.98 MB and 0.51 MB, respectively. We see that a significant space saving is achieved with the subtree-based XML storage.

As for the XPFS tree, it is mandatory in System/T. In System/N, it is optional. (If the path-based XML storage and XPath processing is employed in System/N as described in this paper, the XPFS tree is mandatory even in System/N.) We generated 11 auction.xml documents of various sizes in XMark. For each of them, we measured the size of the XPFS tree implemented as an $n$-ary tree stored as a file. Table 4 shows that compared with the size of the auction.xml source document, the space required for the XPFS tree is negligible.

\section{Related Work}

Much work has been conducted on storing XML data. Several techniques that employ an RDBMS were proposed first [6][8][11][12]. Since the range numbering schemes were proposed in [1][4], the techniques of XML storing have been centered around XML node labeling. As such, the XML node labeling schemes had received much attention, and the schemes ORDPATHS [13], QED [14], and so on were developed. As for XPath processing, the structural join based on XML node labeling has been established as a core technique [1] [2][4]. As we pointed out in this paper, however, the current state-of-the-art technique of XML storing and XPath processing is node-based.

In STORED [6], the irregular parts of the XML data was treated as overflow when the XML data is mapped to relational schema. Such overflow data could be stored as a subtree. In [7], a hybrid design of object-relational schema to store XML data was investigated. The irregular parts and/or infrequently accessed parts of the XML data are stored as subtrees while all the XML nodes in the rest are separately stored. In these work, however, the capability of dealing with the stored subtrees without parsing in query processing was not provided.

In Natix [15] and SystemRX [16], which provide the native XML data management functions, the nodes close to each other (e.g., parent-child) or the nodes in a subtree are put to the same page (in the slotted page structure). In Natix, such subtrees are identified by fragmenting the XML documents with the use of virtual nodes as connector between subtrees. However, those subtrees are stored in some parsed form so that the nodes in them can be accessed. Strictly speaking, they use node-based storage with node clustering capability. In our work, the storage unit is a subtree, and the subtrees are not parsed during XPath processing, and thus, it can be stored as just plain text (byte stream).

The techniques of XPath processing over a stream of XML subtrees fragmented out of a source XML document in a resource-limited client devices were proposed [17][18]. In those techniques, however, each received subtree is parsed in query processing. In [5], active XML processing was investigated where some portions of XML data are dynamically handled as XML subtrees especially in the web service applications. However, the capability of dealing with the subtrees without parsing in query processing was not provided.

Techniques of exploiting XML path schema had received little attention. XRel [8] and XParent [19] are early systems with such a feature. Recently, in [20], a native XML data 
management techniques that exploit the path information called path synopsis with which a very space-efficient XML storage can be achieved were proposed. These work, however, does not feature subtree-based XML storage and XPath processing with the path information. The XPFS tree in our work plays the similar role as the well-known DataGuide [21] or the path synopsis [20] which summarizes the path structure of a semistructured or XML data except that the XPFS tree also specifies the XML fragmentation schema. It also corresponds to a part of the fragment context specification defined as a core component of the XML Fragment Interchange specified by the W3C XML Fragment Working Group [22].

\section{Conclusions and Future Work}

In this paper, we proposed the subtree-based XML storage and XPath processing as a generalization of the state-of-the-art node-based XML storage and XPath processing. The main contributions of this paper are:

1. We proposed the technique of subtree-based XML storing and XPath processing whereby the parsing of the subtrees involved in the query is not required.

2. Through implementation and experiments, we showed that our proposed solution is a feasible generalization of the node-based solution both in time and in space.

As shown in the query performance experiments (Table 3), the frequently requested XML nodes (i.e., hot nodes) are desired to be the roots of the subtrees while the cold ones are placed inside the subtrees. As a future work, we are now considering a problem of optimization of fragmenting an XML document modeled as a tree into subtrees given a set of XPath expressions and their frequencies.

Since the contents of the stored subtrees except for their roots need not be accessed during query processing, another future work we are interested in is the application of the proposed subtree-based XML storage and XPath processing technique to secure XML data processing.

The primary focus in this paper was on the correctness of XPath processing without parsing the stored subtrees involved. However, updatability is also an important issue. The proposed technique should not undermine updatability. As for dynamic XML documents, they can also be supported with the proposed technique. A basic assumption in this paper is that the XML documents conform to some DTD. Also the basic XML fragmentation rule is enforced. It states that the repeating or optional elements specified with,$+{ }^{*}$, or ? in the DTD are to be the roots of different subtrees. As such, each of the nodes that reside inside a subtree (excluding the root) is the node that appear exactly once (at least once as well as at most once) as a subelement or an attribute of its parent. That is, their cardinality (i.e., the number of occurrences) is 1 . Thus, the internal structure of any subtree instance is supposed to remain static, conforming to the schema. In other words, every non-root node in a subtree appears at least once (which means it cannot be deleted) and at most once (which means it is already inserted). Meanwhile, the repeating or optional nodes are the roots of some subtrees, and the entire subtree can be inserted or deleted as a static unit. To support the updates that would change the internal structure of a subtree, schema evolution needs to be considered. Although the types of allowed updates with our technique are restricted to some extent because of the assumption of having DTD and the basic XML fragmentation rule, they cover practical types of updates while conformance to the schema is preserved. Thus, our technique can be applied to a wide range of dynamic environment as well. An important issue requiring further investigation is the relationship among update types, schema evolution, and fragmentation.

Other issues that need to be addressed include transaction management in multi-user environments. As far as locking is concerned, for example, the new types of muti-user 
concurrent operations include the followings: (1) Two users are to read different parts of the same subtree. (2) One is trying to read a node inside a subtree while the other intends to delete the same subtree, and so on. A locking protocol similar to the multiple granularity intent locking (acquiring a weaker lock on the subtree first, and then a stronger one on the nodes inside the subtree) could be devised for concurrency control. In the former case, both users are supposed to be in the shared lock mode, and not in conflict. Both users are required to acquire the shared lock on the subtree. In the latter, they are in conflict, which is detected as follows: With the node-based storage, a node $n$ is uniquely identified with $L_{n}$ (the label of $n$ ) whereas with our subtree-based storage, the same node $n$ which might be residing inside a subtree $t$ is uniquely identified with the pair $\left\langle L_{r}, I_{n}>\right.$ where $L_{r}$ is the label of the root of $t$, and $I_{n}$ is the path ID of $n$. As such, an afore-mentioned intent locking-like protocol can reveal the conflict. Because to obtain a lock on $n$, the lock on $t$ needs to be obtained first, both users are supposed to try to acquire an exclusive lock on $t$ (i.e., the lock on name $L_{r}$ ).

\section{References}

[1] C. Zhang, J. Naughton, D. DeWitt, Q. Luo, and G. Lohman, "On Supporting Containment Queries in Relational Database Management Systems," in Proc. of ACM SIGMOD International Conf. on Management of Data, pp. 425-436, 2001.

[2] S. Al-Khalifa, H. Jagadish, N. Koudas, J. Patel, D. Srivastava, Y. Wu, "Structural Joins: A Primitive for Efficient XML Query Pattern Matching," in Proc. of 18th International Conf. on Data Engineering, pp. 141-152, 2002.

[3] N. Bruno, N. Koudas, and D. Srivastava, "Holistic Twig Joins: Optimal XML Pattern Matching," in Proc. of ACM SIGMOD International Conf. on Management of Data, pp. 310-321, 2002.

[4] Q. Li and B. Moon, "Indexing and Querying XML Data for Regular Path Expressions," in Proc. of 27th International Conf. on Very Large Data Bases, pp. 361-370, 2001.

[5] S. Abiteboul, O. Benjelloun, B. Cautis, I. Manolescu, T. Milo, and N. Preda, "Lazy Evaluation for Active XML," in Proc. of ACM SIGMOD International Conf. on Management of Data, pp. 227-238, 2004.

[6] A. Deutsch, M. Fernandez, D. Suciu, "Storing Semistructured Data with STORED," in Proc. of ACM SIGMOD International Conf. on Management of Data, pp. 431-442, 1999.

[7] M. Klettke and H. Meyer, "XML and Object-Relational Database Systems - Enhancing Structural Mappings based on Statistics," in Proc. of 3rd International Workshop on Web and Databases, pp. 63-68, 2000.

[8] Yoshikawa, T. Amagasa, T. Shimura, S. Uemura, "XRel: A Path-Based Approach to Storage and Retrieval of XML Documents Using Relational Databases," ACM Trans. on Internet Technology, vol. 1, no. 1, pp. 110-141, 2001.

[9] J. Clark and S. DeRose, editors, XML Path Language (XPath) version 1.0, W3C Recommendation, Nov. 1999, http://www.w3.org/TR/xpath.

[10] A. Schmidt, F. Wass, M. Kersten, M. Carey, I. Manolescu, and R. Busse, "XMark: A Benchmark for XML Data Management," in Proc. of 28th International Conf. on Very Large Data Bases, pp. 974-985, 2002.

[11] D. Florescu and D. Kossmann, "Storing and Querying XML Data Using an RDBMS," IEEE Data Engineering Bulletin, vol. 22, no. 3, pp. 27-34, 1999.

[12] J. Shanmugasundaram, K. Tufte, C. Zhang, G. He, D. DeWitt, and J. Naughton, "Relational Databases for Querying XML Documents: Limitations and Opportunities," in Proc. of 25th International Conf. on Very Large Data Bases, pp. 302-314, 1999.

[13] P. O’Neil, E. O’Neil, S. Pal, I. Cseri, G. Schaller, and N. Westbury, “ORDPATHs: Insert-Friendly 
XML Node Labels," in Proc. of ACM SIGMOD International Conf. on Management of Data, pp. 903-908, 2004.

[14] C. Li and T. Ling, "QED: A Novel Quaternary Encoding to Completely Avoid Relabeling in XML Updates," in Proc. of International Conf. on Information and Knowledge Management, pp. 501-508, 2005.

[15] T. Fiebig, S. Helmer, C. Kanne, G. Moerkotte, J. Neumann, R. Schiele, and T. Westmann, “Anatomy of a Native XML Base Management System," VLDB Journal, vol. 11, no. 4, pp. 292-314, 2002.

[16] K. Beyer, R. Cochrane, V. Josifovski, J. Kleewein, G. Lapis, G. Lohman, R. Lyle, F. Ozcan, H. Pirahesh, N. Seemann, T. Truong, B. Van der Linden, B. Vickery, and C. Zhang, "System RX: One Part Relational, One Part XML," in Proc. of ACM SIGMOD International Conf. on Management of Data, pp. 374-358, 2005.

[17] S. Bose and L. Fegaras, "XFrag: A Query Processing Framework for Fragmented XML Data," in Proc. of 8th International Workshop on Web and Databases, pp. 97-102, 2005.

[18] H. Huo, G. Wang, X. Hui, R. Zhou, B. Ning, and C. Xiao, "Effiecient Query Processing for Streamed XML Fragments," in Proc. of 11th International Conf. on Database Systems for Advanced Applications, pp. 468-482, 2006.

[19] H. Jiang, H. Lu, W. Wang, and J. Yu, "XParent: An Efficient RDBMS-based XML Database System,” in Proc. of 18th International Conf. on Data Engineering, pp. 335-336, 2002.

[20] C. Mathis, T. Härder, and K. Schmidt, "Storing and Indexing XML Documents Upside Down," Computer Science - Research and Development, vol. 24, no. 1-2, pp. 51-68, 2009.

[21] R. Goldman and J. Widom, "DataGuides: Enabling Query Formulation and Optimization in Semistructured Databases," in Proc. of 23rd International Conf. on Very Large Data Bases, pp. 436-445, 1997.

[22] P. Grosso and D. Veillard, editors, XML Fragment Interchange (XFI), W3C Candidate Recommendation, Feb. 2001, http://www.w3.org/TR/xml-fragment.

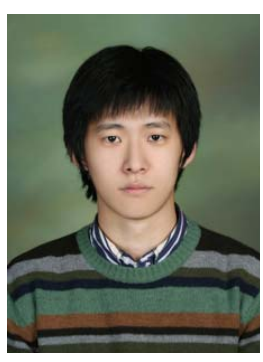

Ki Hoon Shin received the B.E. degree in Computer Science and Engineering from Chung-Ang University, Seoul, Korea in 2009. He is currently a M.S. student in Computer Science and Engineering at Chung-Ang University. His research interests include XML query processing and sensor network database.

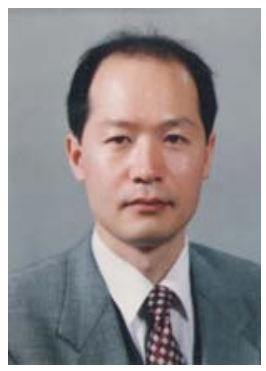

Hyunchul Kang received the B.E. degree in Computer Engineering from Seoul National University, Seoul, Korea in 1983, and received the M.S. and Ph.D. degrees in Computer Science from the University of Maryland, College Park, U.S.A. in 1985 and 1987, respectively. In 1988, he joined the School of Computer Science and Engineering, Chung-Ang University, Seoul, Korea where he is currently a Professor. His current research interests include XML and web data management, mobile data management, and sensor network database. 